\title{
The right thalamus may play an important role in anesthesia- awakening regulation in frogs
}

\author{
Yanzhu Fan ${ }^{1,2}$, Xizi Yue ${ }^{1,3}$, Fei Xue ${ }^{1}$, Steven E. Brauth ${ }^{4}$, Yezhong Tang ${ }^{1}$ ， Guangzhan Fang ${ }^{\text {Corresp. }{ }^{1,3}}$ \\ ${ }^{1}$ Chengdu Institute of Biology, Chinese Academy of Sciences, Chengdu, Sichuan, People's Republic of China \\ 2 University of Chinese Academy of Sciences, Beijing, People's Republic of China \\ 3 College of Life Sciences, China West Normal University, Nanchong, Sichuan, People's Republic of China \\ 4 Department of Psychology, University of Maryland, the United States of America \\ Corresponding Author: Guangzhan Fang \\ Email address: fanggz@cib.ac.cn
}

Background. Previous studies have shown that the mammalian thalamus is a key structure for anesthesia-induced unconsciousness and anesthesia-awakening regulation. However, both the dynamic characteristics and probable lateralization of thalamic functioning during anesthesia-awakening regulation are not fully understood and little is known of the evolutionary basis of the role of the thalamus in anesthesia-awakening regulation.

Methods. An amphibian species, the South African clawed frog (Xenopus laevis) was used in the present study. The frogs were immersed in triciane methanesulfonate (MS222) for general anesthesia. Electroencephalogram (EEG) signals were recorded continuously from both sides of the telencephalon, diencephalon (thalamus) and mesencephalon during the pre-anesthesia stage, administration stage, recovery stage and post-anesthesia stage. EEG data was analyzed including calculation of approximate entropy (ApEn) and permutation entropy (PE).

Results. ApEn and PE values differed significantly between anesthesia stages, with the highest values occurring during the awakening period and the lowest values during the anesthesia period. There was a significant correlation between the duration and ApEn or PE values during anesthesia-awakening cycle primarily for the right diencephalon (right thalamus). ApEn and PE values for females were significantly higher than those for males.

Discussion. ApEn and PE measurements are suitable for estimating depth of anesthesia and complexity of amphibian brain activity. The right thalamus appears physiologically positioned to play an important role in anesthesia-awakening regulation in frogs indicating an early evolutionary origin of the role of the thalamus in arousal and consciousness in land vertebrates. Sex differences exist in the neural regulation of general anesthesia in frogs. 
$1 \quad$ The Right Thalamus may Play an Important Role in AnesthesiaAwakening Regulation in Frogs

3 Yanzhu Fan a,b, Xizi Yue ${ }^{a, c}$, Fei Xue ${ }^{a}$, Steven E. Brauth ${ }^{d}$, Yezhong Tanga ${ }^{a}$, and Guangzhan

$4 \quad$ Fang $^{\mathrm{a}, \mathrm{c}^{*}}$

$5{ }^{a}$ Chengdu Institute of Biology, Chinese Academy of Sciences, No.9 Section 4, Renmin South

6 Road, Chengdu, Sichuan, People's Republic of China

7 bUniversity of Chinese Academy of Sciences, 19A Yuquan Road, Beijing, People's Republic of 8 China

$9{ }^{c}$ College of Life Sciences, China West Normal University, No.1 Shida Road, Nanchong, Sichuan,

People's Republic of China

11 dDepartment of Psychology, University of Maryland, College Park, MD20742, USA

13 * To whom all correspondence should be addressed: Associate Prof. Guangzhan Fang, e-mail:

14 fanggz@cib.ac.cn 
16 Abstract

17 Background. Previous studies have shown that the mammalian thalamus is a key structure for

anesthesia-induced unconsciousness and anesthesia-awakening regulation. However, both the dynamic characteristics and probable lateralization of thalamic functioning during anesthesiaawakening regulation are not fully understood and little is known of the evolutionary basis of the role of the thalamus in anesthesia-awakening regulation.

Methods. An amphibian species, the South African clawed frog (Xenopus laevis) was used in the present study. The frogs were immersed in triciane methanesulfonate (MS222) for general anesthesia. Electroencephalogram (EEG) signals were recorded continuously from both sides of the telencephalon, diencephalon (thalamus) and mesencephalon during the pre-anesthesia stage, administration stage, recovery stage and post-anesthesia stage. EEG data was analyzed including calculation of approximate entropy (ApEn) and permutation entropy (PE).

Results. ApEn and PE values differed significantly between anesthesia stages, with the highest values occurring during the awakening period and the lowest values during the anesthesia period. There was a significant correlation between the duration and ApEn or PE values during anesthesiaawakening cycle primarily for the right diencephalon (right thalamus). ApEn and PE values for females were significantly higher than those for males.

Discussion. ApEn and PE measurements are suitable for estimating depth of anesthesia and complexity of amphibian brain activity. The right thalamus appears physiologically positioned to play an important role in anesthesia-awakening regulation in frogs indicating an early evolutionary origin of the role of the thalamus in arousal and consciousness in land vertebrates. Sex differences 
37 exist in the neural regulation of general anesthesia in frogs. 


\section{Introduction}

40 General anesthesia (GA) is a drug-induced depression of the central nervous system (CNS)

41 that permits long-term operations and experimental studies requiring invasive procedures

42 (Antognini, Barter \& Carstens, 2005; Goddard \& Smith, 2013), which is characterized by

unawareness or unconsciousness, analgesia and immobilization (Brown, Lydic \& Schiff, 2010;

Franks, 2008; Pocock \& Richards, 1993). Previous studies have shown that specific brain regions,

including the midbrain reticular formation, thalamus and some brain regions located within the

parietal and frontal association cortex, are more sensitive to anesthesia than others (Heinke \&

Koelsch, 2005). Furthermore functional-brain-imaging data in humans reveal suppression of

activity in specific brain areas including thalamic nuclei and the midbrain reticular formation

during general anesthesia (Alkire, Haier \& Fallon, 2000). Anesthetics can suppress cortico-

thalamo-cortical activity and produce unconsciousness by reducing membrane excitability in the

thalamo-cortical loop, which has been proposed to play a crucial role in the anesthesia-awakening

cycle (Ries \& Puil, 1999). In particular, many neurons within the thalamus exhibit anatomical and

physiological specializations which support large-scale cerebral dynamics related to consciousness

(Domino, 1968). Thus the thalamus, as an information hub, seems well positioned to function as

a key region for regulation of anesthesia-induced unconsciousness by gating sensory information

processing (Franks, 2008; Ries \& Puil, 1999). Nevertheless, the dynamic properties of the thalamus

during the anesthesia-awakening cycle remain unclear. 
60 2015; Rogers, Vallortigara \& Rogers, 2017; Rosa-Salva et al., 2012; Roussigné, Blader \& Wilson,

61 2012; Vallortigara et al., 1998), and appears to be a fundamental aspect of nervous system

62 organization. Brain lateralization may enable simultaneous channeling of different types of

63 information into lateralized brain circuits thereby enabling separate and parallel processing in the

64 two hemispheres (Dadda et al., 2009; Fang et al., 2014; Rogers \& Vallortigara, 2015; Rogers,

65 Vallortigara \& Andrew, 2013; Vallortigara \& Rogers, 2005). Relatively few neurophysiological

66 studies have focused on whether regulation of general anesthesia is also asymmetric. The purpose

67 of this paper is to investigate the neural mechanisms of general anesthesia in an amphibian species,

68 the South African Clawed frog (Xenopus laevis), an important animal model for developmental

69 and genetic studies (Schultz \& Dawson, 2003), which has been frequently used for basic vertebrate

70 nervous system functioning (Guénette, Giroux \& VAChon, 2013), to test the theory that lateralized

71 thalamic general anesthesia regulation is an evolutionarily conserved feature of land vertebrates.

The electroencephalogram (EEG) reflects the summed post-synaptic potentials generated by

pyramidal cells of the cerebral cortex and can be recorded on the surface of the scalp

74 (Muthuswamy, Roy \& Sharma, 1996). Though the EEG allows assessing brain activity during different anesthesia stages, results of traditional linear analysis based on raw EEG data are difficult to interpret precisely (Billard et al., 1997; Bruhn et al., 2006; Katoh, Suzuki \& Ikeda, 1998; Mahon et al., 2008). This is because neuronal ensembles exhibit important nonlinear behaviors whose characteristics can only be adequately described using nonlinear parameters (Bruhn, Röpcke \& Hoeft, 2000; Burioka et al., 2005). Among these methods, both approximate entropy (ApEn) and permutation entropy (PE) are statistical parameters that can quantify randomness and the 
81

82

83

84

85

86

87

88

predictability of a time series and can be used to depict the complexity of EEG signals and the effects of anesthetic drugs on the central nervous system (Bruhn, Röpcke \& Hoeft, 2000; Fan et al., 2011; Li, Cui \& Voss, 2008; Liang et al., 2015; Ouyang et al., 2010; Pincus, 1991).

The amphibian brain exhibits the same segmental architecture as birds, reptiles and mammals including humans and is composed of the telencephalon, diencephalon, mesencephalon, metencephalon and myelencephalon (Wilczynski \& Endepols, 2007). The diencephalon consists of two main cellular aggregates, the thalamus dorsally and the hypothalamus ventrally, which exhibit the same general patterns of connectivity as amniotes (Butler, 1995; Laberge et al., 2008). Anatomically the diencephalon is caudal to the telencephalon and rostral to the mesencephalon and located below the skull at a point where the cerebrum is disappearing. Thus, it is possible to implant electrodes in the diencephalon and to obtain high signal-to-noise ratio EEG activity originating in the thalamus.

Triciane methane-sulfonate (MS-222), which can block motor activity and nociception through relatively long term blockade of action potential initiation via voltage gated sodium channels in the brain and muscles (Ramlochansingh et al., 2014), is widely used to induce anesthesia for amphibians (Downes, 1995; Lalonde-Robert, Beaudry \& Vachon, 2012). In addition, a previous EEG study demonstrated that MS-222 can lead to profound CNS depression and is capable of causing unconsciousness in X. laevis (Lalonde-Robert et al., 2012). For example, $X$. laevis can be anesthetized effectively with concentrations ranging from 1 to $5 \mathrm{~g} / \mathrm{L}$ MS-222 solution (Torreilles, McClure \& Green, 2009).

This study was conducted on X. laevis frogs with implanted electrodes in the telencephalon, 
102 diencephalon and mesencephalon, respectively. The animals were general anesthetized by 103 immersion in MS-222. EEG signals were obtained during the pre-anesthesia, administration, 104 recovery and post-anesthesia stages and were recorded continuously. The ApEn and PE for each 105 stage were calculated in order to explore the relationships between neural activities recorded in 106 each brain area across the anesthesia-awakening cycle including possible functional lateralization 107 for general anesthesia regulation. 
108 Material and Methods

109 Animal

110 Fourteen South African clawed frogs of both sexes (seven males and seven females) bred in 111 the lab were used in the present study. The subjects were separated by sex and raised in two aquaria $112(120 \times 50 \mathrm{~cm}$ and $60 \mathrm{~cm}$ deep) with water depth approximately $20 \mathrm{~cm}$. The animals were fed every 113 three days and the water was replaced once a week. The aquaria were placed in a room in which 114 the temperature was maintained at $20 \pm 1{ }^{\circ} \mathrm{C}$ with a $12 / 12$ light-dark cycle (lights on at 08:00 h). 115 The subjects measured $8.1 \pm 1.1 \mathrm{~cm}$ (mean $\pm \mathrm{SD}$ ) in body length and $67.1 \pm 22.2 \mathrm{~g}$ in body mass at 116 the time of surgery. All surgery was performed under MS-222 anesthetic, and all efforts were made 117 to minimize discomfort. All animal procedures were carried out in accordance with the Animal 118 Care and Use Committee of Chengdu Institute of Biology, Chinese Academy of Sciences 119 (Approval number: 2016005).

120

121

122

123

124 125

126

127

128

Surgery

All experiments were conducted during April to May (this species breeds between April and September in our lab), 2016. The animals were deeply anesthetized via water bath using $500 \mathrm{ml}$ of MS-222 solution (3.5g/L) and buffered by adding sodium bicarbonate to achieve a neutral $\mathrm{pH}$ (7.0-7.4). The optimum depth of anesthesia for surgery was determined to be when the withdrawal reflex to toe pinching (i.e. the toe pinch response by grasping a digit with the tweezers) is lost (Tasnim, 2016), i.e. responsiveness to pinch limb test is used as a proxy for testing consciousness status. MS-222 solution was wiped to the animal's skin using a cotton swab when it was necessary during surgery. The animals were wrapped with wet cotton gauze. Seven cortical EEG electrodes, 
129

130

131

132

133

134

135

136

137

138

139

140

141

142

143

144

145

146

147

148

149

composed of miniature stainless steel screws $(\varphi 0.8 \mathrm{~mm})$, were inserted in the skull by turning 3.5

rotations to implant at a depth of about $1.1 \mathrm{~mm}$ : the left and right sides of the telencephalon, diencephalon and mesencephalon (LT, RT, LD, RD, LM and RM) and referenced to the electrode above the cerebellum (P) (Fig. 1). Ten seconds of typical EEG waves are presented along with the corresponding electrode pairs in Fig. 1. The electrodes above LT and RT were implanted bilaterally $6.4 \mathrm{~mm}$ anterior to the lambda (i.e. the vertex where the skull sutures intersect) and $1.0 \mathrm{~mm}$ lateral to the midline respectively, and the electrodes above LD and RD were implanted bilaterally 3.4 $\mathrm{mm}$ anterior to the lambda and $1.0 \mathrm{~mm}$ lateral to the midline respectively, while the electrodes above LM and RM were implanted bilaterally $1.4 \mathrm{~mm}$ anterior to the lambda and $1.0 \mathrm{~mm}$ to the midline, respectively. P was implanted $1.0 \mathrm{~mm}$ posterior to the lambda at the midline (Fig. 1). One end of all electrode leads, formvar-insulated nichrome wires, was twined tightly on the screws and fixed on the skull of the frog with dental acrylic, while the other end was soldered to the pins of the light connector. Finally, the skin edges and muscles surrounding the wound were treated with the ointment with triple antibiotic and pain relief (CVS pharmacy, Woonsocket, RI, USA) to prevent infection and discomfort.

Each frog was housed individually for one day for recovery before the following experiments were performed. After the end of all experiments, the subjects were euthanized by immersion in MS-222 solution for a prolonged period of time and the electrode locations were confirmed by injecting hematoxylin dye through the skull holes in which the electrodes had previously been installed (Fig. S1).

\section{Data acquisition}



in which the background noise was $24.3 \pm 0.7 \mathrm{~dB}$ (mean $\pm \mathrm{SD}$ ) within a transparent plastic box with a floor area of $18 \times 11 \mathrm{~cm}^{2}$, an upper cover area of $20 \times 13 \mathrm{~cm}^{2}$ and $12 \mathrm{~cm}$ in height. A sponge $(17 \times 10 \times 1 \mathrm{~cm})$ which had absorbed about $200 \mathrm{ml}$ of water was placed at the bottom of the box.

Lights and temperature in the chamber were maintained as in the housing room. A video camera with an infrared light source and motion detector was appended centrally approximately $40 \mathrm{~cm}$ above the box for monitoring the subject's behavior from outside the chamber.

Before the experiments began the subject was connected to the signal acquisition system stages: (1) During Stage I, the awake animal was placed in the experimental box and free to move for 30 min before anesthesia; in fact the subject usually kept immobility with its head towards one corner of the box; (2) During Stage II, the subject was transferred to another box (of the same size within the experimental chamber) containing a $200 \mathrm{ml}$ MS-222 solution $(3.5 \mathrm{~g} / \mathrm{L})$ with a depth about $1 \mathrm{~cm}$, in which the subject was immersed for about 5 min until the toe pinch response (grasping one of the four frog's digits selected randomly every 30s) disappeared, and then subsequently removed quickly from the MS-222 solution; (3) Stage III was defined as the time period between returning the subject to the experimental box and the moment locomotor activity first reappeared while one of the digits was selected randomly to touch every 30 s by a cotton swab; after anesthesia.

\section{Approximate entropy (ApEn)}


172 (Pincus, 1991), which is particularly effective for analyzing short and noisy time-series data and

which can categorize a wide variety of systems ranging from multi periodic, stochastic to mixed

Shafer, 2001; Bruhn, Röpcke \& Hoeft, 2000; Bruhn et al., 2000; Fan et al., 2011; Hudetz, 2002;

Hudetz, Wood \& Kampine, 2003; Koskinen et al., 2006; Kreuzer et al., 2010; Noh et al., 2006;

Sleigh et al., 2004). The procedure for estimating ApEn is described as follows:

For a given time series $u(i), i=1, \ldots, N$ from measurements equally in time, form a sequence

of vectors that are defined by

$$
X(i)=[u(i), u(i+1), \ldots, u(i+m-1)], i=1, \ldots, N-m+1
$$

where $m$ is the embedding dimension of phase space. The distance between vectors $X(i)$ and $X(j)$ can be defined as

$$
d[X(i), X(j)]=\max _{k=1,2, \ldots, m}|u(i+k-1)-u(j+k-1)|
$$

For a given $i \leq N-m+1, N^{m}(i)$ is the number of $j$ in dimension $m$ such that $d[X(i), X(j)]$ $\leq r$, then $C_{i}^{m}(r)$ is defined as

$$
C_{i}^{m}(r)=(N-m+1)^{-1} N^{m}(i)
$$

where $r$ is the tolerance (i.e. previous setting of minimal distance between vectors $X(i)$ and $X(j)$ ). 
191 Next step is to compute the natural logarithm of each $C_{i}^{m}(r)$ and average it over $i$

192

$$
\emptyset^{m}(r)=(N-m+1)^{-1} \sum_{i=1}^{N-m+1} \ln C_{i}^{m}(r)
$$

193

Then increase the embedding dimension, i.e. from $m$ to $m+1$. Repeat steps (3) (4) to obtain

Finally, ApEn can be calculated as

$$
\operatorname{ApEn}(m, r, N)=\phi^{m}(r)-\phi^{m+1}(r)
$$

Mathematically, ApEn measures the likelihood that runs of patterns which are close for $m$

observations will remain close on the following incremental comparisons (Jaušovec \& Jaušovec,

2010). Thus, ApEn is a non-negative number calculated from a time series using the above

protocols. Smaller values of ApEn imply a stronger regularity or persistence in the time series

while larger values indicate greater fluctuation or irregularity. Usually the parameter $m$ is set to 1

or 2 ( $m=2$ recommended) while $r$ can range from 0.1 to 0.25 times the $\mathrm{SD}$ of the original data

sequence (Pincus, 1991).

\section{Permutation Entropy (PE)}

Bandt and Pompe recently proposed a new permutation method to map a continuous time

series onto a symbolic sequence, where the statistics of the symbolic sequences are called

permutation entropy (PE) (Bandt \& Pompe, 2002). PE is an appropriate complexity measure for

chaotic time series, in particular in the presence of dynamical and observational noise. The

advantages of PE are its simplicity, extremely fast calculation, its robustness and invariance with 
Given a time series $x_{t}(t=1,2,3 \ldots)$, an embedding procedure forms vectors

$212 X_{t}\left[x_{t}, x_{t+\tau}, \ldots x_{t+m \tau}\right]$ with the embedding dimension $m$ and the lag $\tau$. The vector $X_{t}$ can be arranged

213 in an increasing order. For different $m$, there will be $m$ ! possible order patterns, which are also

214 called permutations. Considering each permutation as a symbol, the vectors $X_{t}$ can be represented

215 by a symbol sequence; the distinct number of symbols $(J)$ should be less than or equal to $m$ !,

216 namely $J \leq m$ ! For the time series $x_{t}$, the probability distributions of the distinct symbols are

217 defined as $p_{1}, p_{2}, \ldots p_{j}$; the PE of this time series is defined by

$218 \quad H_{p}(m)=-\sum_{j=1}^{J} p_{j} \ln p_{j}$

219 The corresponding normalized entropy can be defined as follows:

$$
H_{p}=H_{p}(m) / \ln (m !)
$$

The largest value of $H_{p}$ is one, meaning the time series is completely random; while the

smallest value of $H_{p}$ is zero, meaning the time series is very regular. In short, the permutation

entropy refers to the local order structure of the time series, which can give a quantitative complexity measure for a dynamical time series. for EEG recordings. For practical purposes, $m=3, \ldots, 7$ is recommended (Bandt \& Pompe, 2002), and that for a long EEG recording, a large value of $m$ is better (Li, Cui \& Voss, 2008). On the other hand, the length of the EEG recording should be larger than $m$ ! in order to achieve a proper differentiation between deterministic and stochastic dynamics (Li et al., 2014; Ouyang et al., 2010). In addition, to allow every possible order pattern of dimension $m$ to occur in a time series 
232 of length $N$, the condition $m ! \leq N-(m-1) \tau$ must hold. Moreover, $N \gg m !+(m-1) \tau$ is required to

233 avoid undersampling (Amigó, Zambrano \& Sanjuán, 2007). For this reason, given $m$ dimensions,

234 we need to choose $N \gg(m+1)$ !. Since this study concentrates on the detection of dynamical

235 changes in the EEG recording, too large a value of $m$ or $N$ would be inappropriate. Therefore $m=$

2365 and $N=1000$ were used for calculating PE in the light of previous studies (Li, Cui \& Voss, 2008;

237 Liang et al., 2015; Ouyang et al., 2010).

238

239

240

241

242

243

244

245

246

247

248

249

250

251

252

\section{Data processing}

In order to evaluate the appropriate parameters for ApEn, five minutes of EEG data during Stage I were selected randomly. After band-pass filtering (0.5-45 Hz) and downsampling at 256 $\mathrm{Hz}, r$ and $N$ were determined by calculating ApEn $(m, r, N)$ with increasing $r$ from 0.1 to $0.4 \mathrm{SD}$ in steps of 0.05 and $N$ from 100 to 2000 in steps of 100 for all randomly selected EEG segments while $m=2$. ApEn reached its maximum on a plateau when $N=500$ and this plateau was stable only when $r=0.15 \mathrm{SD}$. Therefore, in the present study, ApEn for EEG data was computed using a slide window of $N=512$ (2 s of the EEG signal) overlapping $N / 2$ for each step while $r=0.15 \mathrm{SD}$, to yield an ApEn vector. Furthermore, in accordance with previous studies (Li, Cui \& Voss, 2008; Liang et al., 2015; Ouyang et al., 2010), each epoch with $N=1000$ (i.e. 10 s of the EEG signal) overlapping $\mathrm{N} / 2$ was used after band-pass filtering $(0.5-45 \mathrm{~Hz})$ and downsampling at $100 \mathrm{~Hz}$ for calculating PE.

Any epoch with an amplitude extremum beyond $\pm 100 \mu \mathrm{v}$ was discarded as artifact. The designation of artifact in one channel resulted in removal of data in all other channels in order to ensure that datasets derived from all channels were derived from the same time periods. ApEn or 
253 PE was then averaged for each stage, each channel and each subject based on artifact-free epochs.

\section{Statistical analyses}

The normality of the distribution and homogeneity of variance for ApEn and PE values were estimated with the Shapiro-Wilk $W$ test and Levene's test, respectively. ApEn and PE values were statistically analyzed using a three-way repeated measured ANOVA with the variables of "sex"

258 (male/female), "stage" (Stages I, III and IV, Stage II was not included because of too many artifacts), and "brain area" (LT, RT, LD, RD, LM and RM). Both main effects and interactions were examined. For significant ANOVAs, data were further analyzed for multiple comparisons using the least-significant difference (LSD) test. Greenhouse-Geisser epsilon $(\varepsilon)$ values were employed when the Greenhouse-Geisser correction was necessary. Estimations of the effect size

for ANOVAs were determined with partial $\eta^{2}$ (partial $\eta^{2}=0.20$ is a small effect size, 0.50 is a medium effect size and 0.80 is a large effect size). Furthermore, Spearman's correlation test was used to calculate the correlation between ApEn or PE values and stage durations. In addition, the Mann-Whitney U-test was used to compare stage durations between sexes for Stages II and III, respectively. SPSS software (release 21) was utilized for the statistical analysis. A significance level of $p<0.05$ and a high significance level of $p<0.001$ were used for all comparisons. 
269

270

271

272

273

274

275

276

277

278

279

280

281

282

283

284

285

286

287

288

289

\section{Results}

\section{ApEn and PE values in different anesthesia-awakening stages}

For ApEn values, ANOVA analysis (Table 1) revealed significant main effects for the factors

“stage" $\left(F_{2,24}=25.127 ; p=0.000 ;\right.$ partial $\left.\eta^{2}=0.677\right)$ and "sex" $\left(F_{1,12}=5.033 ; p=0.045\right.$; partial $\left.\eta^{2}=0.295\right)$ but marginally non-significant effect for the factor "brain area" $\left(F_{5,60}=2.373 ; p=\right.$ 0.050; partial $\eta^{2}=0.165$ ), but no significant interaction between factors was observed. ApEn values in Stage III (the recovery stage) were significantly lower than those in both Stages I (the awake stage) and IV (post-anesthesia stage) $(p<0.05)$, however there was no significant difference between Stages I and IV (Table 1 and Fig. 2). In addition, ApEn values for females were significantly higher than the values for males (Table 1).

For PE values, ANOVA analysis (Table 2) showed significant main effects for the factors “stage" $\left(F_{2,24}=10.489 ; p=0.001 ;\right.$ partial $\left.\eta^{2}=0.466\right)$, "sex" $\left(F_{1,12}=7.895 ; p=0.016 ;\right.$ partial $\eta^{2}$ $=0.397)$ and "brain area" $\left(F_{5,60}=10.584 ; p=0.000\right.$; partial $\left.\eta^{2}=0.469\right)$. Similar to ApEn, PE values in Stage III (the recovery stage) were significantly lower than those in both in Stages I (the awake stage) and IV (post-anesthesia stage) $(p<0.05)$, however there was no significant difference between Stages I and IV (Table 2 and Fig. 2). Similarly, PE values for females were significantly higher than PE values for males (Table 2). In addition, PE values for the right mesencephalon were significantly higher than those for other brain areas, while PE values for both sides of the diencephalon and the left mesencephalon were significantly higher than those for the left telencephalon, and that PE values for the right diencephalon were significantly higher than those for the right telencephalon (Table 2). 
$291 \operatorname{PE}\left(F_{10,120}=6.665 ; p=0.000 ;\right.$ partial $\eta^{2}=0.357$; Table 2$)$. Simple effects analysis showed that

292 PE values for the right mesencephalon during a given stage were significantly higher than those

293 for other brain areas $\left(F_{5,65}=6.583, \varepsilon=0.480, p=0.003\right.$, partial $\eta^{2}=0.336$ in Stage I; $F_{5,65}=8.642$,

$294 p=0.000$, partial $\eta^{2}=0.399$ in Stage III; $F_{5,65}=13.395, p=0.000$, partial $\eta^{2}=0.507$ in Stage IV;

295 Table 3) although the difference between the left and right mesencephalon did not reach statistical

296 significance. In addition, PE values for the right diencephalon during Stage III (the recovery stage)

297 were significantly higher than those for both sides of the telencephalon while PE values for the left

298 diencephalon during stages I and IV were significantly higher than values for the left

299 telencephalon. For each brain area, PE values for Stages I and IV were significantly higher than

300 that in Stage III $\left(F_{2,26}=13.942, p=0.000\right.$, partial $\eta^{2}=0.517$ for the left telencephalon; $F_{2,26}=$

$30112.234, p=0.000$, partial $\eta^{2}=0.485$ for the right telencephalon; $F_{2,26}=10.603, p=0.000$, partial

$302 \eta^{2}=0.449$ for the left diencephalon; $F_{2,26}=5.926, p=0.008$, partial $\eta^{2}=0.313$ for the right

303 diencephalon; $F_{2,26}=9.289, p=0.001$, partial $\eta^{2}=0.417$ for the left mesencephalon; $F_{2,26}=6.507$,

$304 p=0.005$, partial $\eta^{2}=0.334$ for the right mesencephalon; Table 3 ), although the difference between

305 Stages III and IV did not reach statistical significance for the right diencephalon. Moreover, PE

306 values for Stage I was higher than that in Stage III for both sides of the telencephalon and the left

307 diencephalon.

308 Stage durations vs. ApEn or PE values

309 Correlation analysis was used to determine whether the durations of the anesthesia stages

310 (Stages II and III) were associated with ApEn or PE values for each brain area and each stage. For 
311

312

314

315

316

317

318

319

320

321

322

323

324

325

326

327

328

ApEn measurement, significant correlations between stage durations and ApEn values were found exclusively for the right hemisphere, especially for the right thalamus (Table 4 and Fig. 3). ApEn values in Stage I were positively correlated with the duration of Stage II for the right diencephalon, i.e. the right thalamus ( $r=0.534, p=0.049$; Table 4 and Fig. $3 \mathrm{~A})$, while ApEn values in Stage III were negatively correlated with the duration of Stage III for the right hemisphere $(r=-0.591, p=$ 0.026 for the right telencephalon; $r=-0.600, p=0.023$ for the right thalamus; $r=-0.552, p=0.041$ for the right mesencephalon; Table 4 and Fig. 3B). Moreover, ApEn values in Stage IV were negatively correlated with the duration of Stage III for the right thalamus $(r=-0.609, p=0.021$; Table 4 and Fig. 3C). Similar to ApEn, significant correlations between stage durations and PE values were found exclusively for right brain areas (Table 5 and Fig. 4). PE values in Stage II were negatively correlated with the duration of Stage III for the right thalamus and the right mesencephalon $(r=-0.670, p=0.009$ for the former; $r=-0.543, p=0.045$ for the latter; Table 5 and Fig. 4A), while PE values in Stage III were negatively correlated with the duration of Stage III for the right hemisphere $(r=-0.578, p=0.030$ for the right telencephalon; $r=-0.543, p=0.045$ for the right thalamus; $r=-0.675, p=0.008$ for the right mesencephalon; Table 5 and Fig. 4B). In addition, the duration of the administration stage for females was significantly longer than that for males $(\mathrm{U}=7, p=0.025$; Table 6$)$, while the duration of the recovery stage for female was shorter than that for males $(\mathrm{U}=13, p=0.142$; Table 6$)$. 


\section{Discussion}

331

332

333

334

335

336

337

338

339

340

341

342

343

344

345

346

347

348

349

350

\section{Anesthesia-awakening cycle in ApEn or PE variation}

During the awake period (Stage I) animals remain vigilant. Consistent with this the highest

ApEn or PE values reflect the demands placed on the brain for processing internal and external stimuli accurately. During the administration stage (Stage II), the animals gradually became motionless and ApEn or PE values decreased sharply (Fig. 2). At the point that ApEn or PE decreased to the lowest level the animals became apparently unconscious insofar as they exhibited no response to the limb pinching stimulus. During the recovery stage (Stage III) ApEn and PE values increased gradually and the toe pinch response reappeared.

ApEn and PE typically reflects the complexity and regularity of brain activity (Burioka et al., 2005; Liang et al., 2015). High ApEn or PE values indicate high complexity, random and unpredictable changes, whereas low ApEn or PE values indicate low complexity, regularity and predictability of EEG signals (Burioka et al., 2005). In this study, we analyzed changes in the ApEn and PE of EEG signals during the "pre-anesthesia, administration, recovery, and postanesthesia stages" cycle in $X$. laevis frogs, and found that there were significant differences in ApEn or PE among these stages. ApEn and PE values were the highest when awake (pre-anesthesia and post-anesthesia stages) and lowest during the administration stage (Tables 1 and 2; Fig. 2).

The occurrence of the highest ApEn or PE values during the stages the frogs were awake is consistent with the idea that at this time complex tasks (such as accurate perception and the processing of internal and external stimuli) can be carried out by the brain (Heinke \& Koelsch, 2005). EEG ApEn and PE show a sharp decrease around the point of loss-of-responsiveness during 
351 the administration stage, consistent with previous studies in humans reporting that the transition

352 into anesthetic unconsciousness is associated with dramatic and abrupt changes in the population-

353 average membrane voltage in cortical neurons (Heinke \& Koelsch, 2005). The present results

354 indicate that both ApEn and PE values reflect changes in the complexity of amphibian brain

355 activities similar to those found in human patients (Bruhn, Röpcke \& Hoeft, 2000) across different

356 stages in the anesthesia-awakening cycle.

357 Right thalamus may play an important role in anesthesia-awakening regulation

358 Both ApEn and PE can track EEG changes associated with different anesthesia states

359 although PE performs best; ApEn performs best in detecting neuron burst suppression (Liang et

al., 2015). Consistent with this, the main effects of the present results were similar for ApEn and

PE. In the present study, different anesthesia states manifested as behavioral changes that would

be expected to be closely related to neuron burst suppression, a phenomenon that would

physiologically reflect possible functional lateralization for general anesthesia regulation. For this

364

365

366

367

368

369

370

371 reason, the following discussion is mainly based on the ApEn results.

During Stage I (the pre-anesthesia stage), ApEn values in the right thalamus were positively correlated with the duration of Stage II (the administration stage). This means that as the complexity of right thalamic neural network activity increases during the pre-anesthesia stage, induction time for general anesthesia increases. Since the complexity of the EEG represents the activity level of specific brain regions, this correlation shows that the right thalamus exhibits significant EEG pattern changes during the anesthesia cycle. The results also show an inverse correlation between right thalamic ApEn values in Stage IV and the duration of Stage III. This 
372 means that increased inhibition of right hemispheric neural information processing activity results

373 in longer recovery times and that correspondingly, longer recovery times are associated with more

374 inhibited thalamic activity in the post-anesthesia stage. In addition, there was an inverse correlation

375 between right hemispheric ApEn values in Stage III and the duration of this stage, suggesting that

376 maintenance of higher activities in the right hemisphere (including the right thalamus) during the

377 recovery stage increases the likelihood that the subject will easily awaken. It is therefore notable

378 that the significant correlations between EEG ApEn and PE values during the anesthesia-

379 awakening cycle appeared primarily for the right diencephalon in the present study (Tables 4 and

5; Figs. 3 and 4). Thus, it seems reasonable to hypothesize that the right thalamus plays a key

regulatory role in the anesthesia-awakening cycle.

The above described right-lateralized effects could not have resulted from the fact that the

habenular nuclei in amphibians are markedly asymmetrical in size insofar as the left habenular

complex consists of two distinct nuclei whereas the right habenular nucleus consists of only one cell group (Schmidt, 1976). The habenular nucleus is a small bilateral structure, located in the anterior dorsal diencephalon (i.e. the epithalamus), behind the pineal gland, on either side of the third ventricle (Kemali \& Braitenberg, 1969). In the present study, the two diencephalic electrodes were situated immediately above the dorsal thalamus which is the main source of subcortical inputs to the cerebral cortex (Harris, Guglielmotti \& Bentivoglio, 1996). In other words, asymmetric habenular nuclei could not be responsible for the right-lateralized effects observed in the present results, consistent with the idea that the right thalamus is of key importance in anesthesiaawakening regulation in frogs. 
regions which also differ in sensitivity to anesthetics (Heinke \& Koelsch, 2005). Several sites in

the brain including the cerebral cortex, thalamus, limbic system and reticular formation have been

proposed to play key roles in the regulation of consciousness with the thalamo-cortical circuitry as

critically important for controlling consciousness and attention (Heinke \& Koelsch, 2005; Heinke

\& Schwarzbauer, 2002). The thalamus serves as a relay or a gate for much of the sensory

information projected to the telencephalon including the cortico-thalamo-cortical pathway

(Béhuret et al., 2013; Sherman \& Guillery, 2002). Thus as a center of the brain, the thalamus has

been characterized as a compact 'miniature map' of the rest of the brain (Ward, 2011). Consistent

with this idea, thalamic lesions can result in profound cognitive disorders including delirium,

aphasias, confusion, unconsciousness and even death (Llinas et al., 1998; Ward, 2013). Recent

studies using PET and fMRI support the idea that the thalamus functions as a macroscopic locus

(target) in which general anesthetics bring about the unconscious state in patients (Heinke \&

Koelsch, 2005) and which is normally involved in regulating cortical arousal and the activities of

cortical networks (McCormick \& Bal, 1997; Schiff, 2008; Steriade, 1996). Moreover, anesthetic-

induced unconsciousness is consistently associated with a reduction in metabolism or blood flow

in the thalamus, which indicates that the thalamus can act as a consciousness switch (Alkire et al.,

1997; Fiset et al., 1999). Our results suggest that the thalamus is the crucial region for anesthesia-

induced unconsciousness in frogs and regulates the amphibian anesthesia-awakening cycle, similar

412 to the condition in humans (Xie et al., 2011).

413 Although the role of the thalamus in conveying sensory input to the telencephalon is firmly 
414 established in reptiles (Pritz, 2016), the cortico-thalamo-cortical loop remains to be identified.

415 However, human studies have shown that the loop modulates unconsciousness under general

416 anesthetics (Fiset et al., 1999) because arousal is elicited by the brainstem-thalamus-cortex

417 activating system (Steriade, 1996). As a central node of these brain networks, the thalamus plays

418 an important role in supporting consciousness in two ways. First, specific thalamic nuclei relay

419 sensory and motor messages that may become part of the content of consciousness. Second,

420 nonspecific thalamic nuclei are likely involved in the control of cortical arousal originating from

421 the brainstem reticular formation (Zhou et al., 2011).

422

The present results show that the right thalamus is physiologically positioned to play a

regulatory role in anesthesia-awakening cycle. This is consistent with the idea that both the

structural and functional lateralization of nervous system function is conserved throughout

vertebrates including humans (Rogers \& Vallortigara, 2015; Samara \& Tsangaris, 2011;

Vallortigara \& Versace, 2017) and is manifest in many invertebrates. Examples include

preferential use of the right or left hemispheres during various information processing tasks and

behaviors in humans, primates, birds, reptiles, amphibians, fish, bees, fruit flies and nematodes

429 (Fang et al., 2015; Frasnelli, Vallortigara \& Rogers, 2012; Lippolis et al., 2002; Robins \& Rogers,

2006; Rogers, 2014; Rogers et al., 2013; Rosa-Salva et al., 2012; Roussigné, Blader \& Wilson,

2012; Vallortigara et al., 1998; Vallortigara \& Versace, 2017; Xue et al., 2015). For example, the

right hemisphere is usually dominant for spatial attention in humans, while the left preferentially

processes language and formal reasoning (Geschwind \& Miller, 2001). Brain asymmetry is thought

to be a conserved and fundamental feature which enhances the efficiency of information 
435

436

437

438

processing, so that functional specialization of one hemisphere frees the contralateral hemisphere to perform other tasks (Rogers \& Vallortigara, 2015; Vallortigara \& Rogers, 2005).

Brain anatomical asymmetries are the basis of functional lateralization (Samara \& Tsangaris, 2011). The right thalamus is larger than the left in humans (Péran et al., 2009; Sullivan et al., 2004). Moreover, lesions of the left and right thalamus typically do not result in the same language deficits (Ojemann, 1977). Thus, it seems reasonable to speculate that the right thalamus would be less likely to be suppressed by general anesthetics. This speculation is consistent with our current results showing that both ApEn and PE values in the right thalamus were higher than the left counterpart during the awakening period and anesthesia period, although these differences did not reach statistical significance. In other words, the dynamic neural activity changes in the right thalamus would be expected to be smaller than those in the left during anesthesia, consistent with its possible regulation function in the anesthesia-awakening cycle. However, it remains for future research to explicate the causal basis of the right thalamic regulation function including the anatomical and neurophysiological characteristics underlying its apparently important role.

\section{Sex differences in general anesthesia}

The current results show that both ApEn and PE values for females are higher than those for males, the duration of the administration stage for females is significantly longer than males, while the duration of the recovery stage for females is shorter than males. These results indicate that female frogs are less sensitive to the hypnotic effect of anesthetics than males, consistent with clinical studies in humans which have shown that women exhibit higher bispectral index values (Buchanan, Myles \& Cicuttini, 2011) than men under the same anesthetic dosage and usually show 
456 faster recovery after the administration of anesthetics compared with men (Buchanan, Myles \&

457 Cicuttini, 2011; Buchanan et al., 2006).

458 Sexually dimorphic behaviors in mammals typically reflect sex differences in the nature of

459

460

461

462

463

464

465

466

467

468

469

470

471

472

473

474

475

476

reciprocal influences among genes, gonadal functions, hormones and environmental factors

(Kelly, Ostrowski \& Wilson, 1999). Some sex hormones are neurosteroids with anesthetic

properties. For instance estradiol and progesterone are involved in general anesthesia regulation

and post-anesthesia recovery (Buchanan et al., 2006). High doses of progesterone can anesthetize

animals and humans (Bitran, Purdy \& Kellog, 1993; Merryman et al., 1954). Previous research

suggests that progesterone and estradiol affect anesthesia maintenance and recovery by influencing

the excitability of neurons in the brain and brainstem (Woolley \& Schwartzkroin, 1998). Thus, the general anesthesia state depends on the interactions of anesthetics with different receptors in the brain, and sex differences in general anesthesia and post-anesthesia recovery in many animal species may be related to the levels of sex hormones and their distribution (Buchanan, Myles \& Cicuttini, 2011). It thus seems reasonable to speculate that these phenomena include amphibians as well.

On the other hand, previous studies in fishes and amphibians have found that larger subjects need longer MS-222 induction times and exhibit shorter recovery times (Cecala \& Price, 2007; Paduano et al., 2013; Zahl et al., 2009; Zahl et al., 2011). In the present study, the body mass of females on average was twice that of males, suggesting that the observed sex differences may have resulted from body mass differences. Future research is required to determine whether the combined actions of sex hormones and body mass differences bring about the sex differences in 
477 general anesthesia observed here.

478 In summary, both ApEn and PE measurements are suitable for estimating the complexity of 479 amphibian brain activity, the right thalamus appears well positioned physiologically to play an 480 important role in anesthesia-awakening regulation in frogs, and sex differences exist in the neural 481 regulation of general anesthesia in frogs. 


\section{Acknowledgements}

483

The authors gratefully acknowledge all the members of the Behavioral Neuroscience Group

484 for their discussion and help for the experiments reported here. 
485

486

487

488

489

490

491

492

493

494

495

496

497

498

499

500

501

502

503

504

505

506

507

508

509

510

511

512

513

514

515

516

517

518

519

520

521

522

523

524

\section{References}

Alkire MT, Haier RJ, and Fallon JH. 2000. Toward a unified theory of narcosis: brain imaging evidence for a thalamocortical switch as the neurophysiologic basis of anesthetic-induced unconsciousness. Consciousness and Cognition 9:370-386. DOI 10.1006/ccog.1999.0423

Alkire MT, Haier RJ, Shah NK, and Anderson CT. 1997. Positron emission tomography study of regional cerebral metabolism in humans during isoflurane anesthesia. Anesthesiology 86:549-557. DOI 10.1097/00000542199703000-00006

Amigó JM, Zambrano S, and Sanjuán MA. 2007. True and false forbidden patterns in deterministic and random dynamics. EPL (Europhysics Letters) 79:50001

Antognini JF, Barter L, and Carstens E. 2005. Movement as an index of anesthetic depth in humans and experimental animals. Comparative Medicine 55:413-418

Bandt C, and Pompe B. 2002. Permutation entropy: a natural complexity measure for time series. Physical Review Letters 88:174102

Béhuret S, Deleuze C, Gomez L, Frégnac Y, and Bal T. 2013. Cortically-controlled population stochastic facilitation as a plausible substrate for guiding sensory transfer across the thalamic gateway. PLoS Computational Biology 9:e1003401. DOI 10.1371/journal.pcbi.1003401

Billard V, Gambus PL, Chamoun N, Stanski DR, and Shafer SL. 1997. A comparison of spectral edge, delta power, and bispectral index as EEG measures of alfentanil, propofol, and midazolam drug effect. Clinical Pharmacology and Therapeutics 61:45-58. DOI 10.1016/S0009-9236(97)90181-8

Bitran D, Purdy RH, and Kellog CK. 1993. Anxiolytic effect of progesterone is associated with increases in cortical alloprenanolone and GABA A receptor function. Pharmacology Biochemistry and Behavior 45:423-428. DOI 10.1016/0091-3057(93)90260-Z

Brown EN, Lydic R, and Schiff ND. 2010. General anesthesia, sleep, and coma. New England Journal of Medicine 363:2638-2650. DOI 10.1056/NEJMra0808281

Bruhn J, Bouillon T, and Shafer S. 2001. Onset of propofol-induced burst suppression may be correctly detected as deepening of anaesthesia by approximate entropy but not by bispectral index. British Journal of Anaesthesia 87:505-507. DOI 10.1093/bja/87.3.505

Bruhn J, Myles P, Sneyd R, and Struys M. 2006. Depth of anaesthesia monitoring: what's available, what's validated and what's next? British Journal of Anaesthesia 97:85-94. DOI 10.1093/bja/ael120

Bruhn J, Röpcke H, and Hoeft A. 2000. Approximate entropy as an electroencephalographic measure of anesthetic drug effect during desflurane anesthesia. Anesthesiology 92:715-726. DOI 10.1097/00000542-20000300000016

Bruhn J, Röpcke H, Rehberg B, Bouillon T, and Hoeft A. 2000. Electroencephalogram approximate entropy correctly classifies the occurrence of burst suppression pattern as increasing anesthetic drug effect. Anesthesiology 93:981-985. DOI 10.1097/00000542-200010000-00018

Buchanan FF, Myles PS, and Cicuttini F. 2011. Effect of patient sex on general anaesthesia and recovery. British Journal of Anaesthesia 106:832-839. DOI 10.1093/bja/aer094

Buchanan FF, Myles PS, Leslie K, Forbes A, and Cicuttini F. 2006. Gender and recovery after general anesthesia combined with neuromuscular blocking drugs. Anesthesia \& Analgesia 102:291-297. DOI 10.1213/01.ANE.0000181321.55422.C6 
Burioka N, Miyata M, Cornélissen G, Halberg F, Takeshima T, Kaplan DT, Suyama H, Endo M, Maegaki Y, and Nomura T. 2005. Approximate entropy in the electroencephalogram during wake and sleep. Clinical EEG and Neuroscience 36:21-24. DOI 10.1177/155005940503600106

Butler AB. 1995. The dorsal thalamus of jawed vertebrates: a comparative viewpoint. Brain Behavior and Evolution 46:209-223

Cecala KK, and Price SJ. 2007. A comparison of the effectiveness of recommended doses of MS-222 (tricaine methanesulfonate) and Orajel (benzocaine) for amphibian anesthesia. Herpetological Review 38:63-66

Dadda M, Zandonà E, Agrillo C, and Bisazza A. 2009. The costs of hemispheric specialization in a fish. Proceedings Royal Society of London Series B Biological Sciences 276:4399-4407. DOI 10.1098/rspb.2009.1406

Domino EF. 1968. Neuronal mechanisms of ketamine-induced anesthesia. International Journal of Neuropharmacology 7:557-573. DOI 10.1016/0028-3908(68)90067-1

Downes U. 1995. Tricaine methanesulfonate in amphibian: a review. Bull Assoc Rept Amph Vet 5:11-16

Fan SZ, Yeh JR, Chen BC, and Shieh JS. 2011. Comparison of EEG approximate entropy and complexity measures of depth of anaesthesia during inhalational general anaesthesia. Journal of Medical and Biological Engineering 31:359-366. DOI 10.5405/jmbe.820

Fang G, Yang P, Xue F, Cui J, Brauth SE, and Tang Y. 2015. Sound Classification and Call Discrimination Are Decoded in Order as Revealed by Event-Related Potential Components in Frogs. Brain Behavior and Evolution 86:232-245. DOI 10.1159/000441215

Fang GZ, Xue F, Yang P, Cui JG, Brauth SE, and Tang YZ. 2014. Right ear advantage for vocal communication in frogs results from both structural asymmetry and attention modulation. Behavioural Brain Research 266:7784. DOI 10.1016/j.bbr.2014.02.042

Fiset P, Paus T, Daloze T, Plourde G, Meuret P, Bonhomme V, Hajj-Ali N, Backman SB, and Evans AC. 1999. Brain mechanisms of propofol-induced loss of consciousness in humans: a positron emission tomographic study. Journal of Neuroscience 19:5506-5513

Franks NP. 2008. General anaesthesia: from molecular targets to neuronal pathways of sleep and arousal. Nature Reviews Neuroscience 9:370-386. DOI 10.1038/nrn2372

Frasnelli E, Vallortigara G, and Rogers LJ. 2012. Left-right asymmetries of behaviour and nervous system in invertebrates. Neuroscience \& Biobehavioral Reviews 36:1273-1291. DOI 10.1016/j.neubiorev.2012.02.006

Geschwind DH, and Miller BL. 2001. Molecular approaches to cerebral laterality: development and neurodegeneration. American Journal of Medical Genetics 101:370-381. DOI 10.1002/10968628(20010715)101:4

Goddard N, and Smith D. 2013. Unintended awareness and monitoring of depth of anaesthesia. Continuing Education in Anaesthesia Critical Care \& Pain 13:213-217. DOI 10.1093/bjaceaccp/mkt016

Guénette SA, Giroux M-C, and VAChon P. 2013. Pain perception and anaesthesia in research frogs. Experimental Animals 62:87-92. DOI 10.1538/expanim.62.87

Harris JA, Guglielmotti V, and Bentivoglio M. 1996. Diencephalic asymmetries. Neuroscience \& Biobehavioral Reviews 20:637-643. DOI 10.1016/0149-7634(95)00077-1

Heinke W, and Koelsch S. 2005. The effects of anesthetics on brain activity and cognitive function. Current Opinion in Anaesthesiology 18:625-631. DOI 10.1097/01.aco.0000189879.67092.12

Heinke W, and Schwarzbauer C. 2002. In vivo imaging of anaesthetic action in humans: approaches with positron emission tomography (PET) and functional magnetic resonance imaging (fMRI). British Journal of 
566

567

568

569

570

571

572

573

574

575

576

577

578

579

580

581

582

583

584

585

586

587

588

589

590

591

592

593

594

595

596

597

598

599

600

601

602

603

604

605

606

Anaesthesia 89:112-122. DOI 10.1093/bja/aef155

Hudetz AG. 2002. Effect of volatile anesthetics on interhemispheric EEG cross-approximate entropy in the rat. Brain Research 954:123-131. DOI 10.1016/S0006-8993(02)03358-9

Hudetz AG, Wood JD, and Kampine JP. 2003. Cholinergic reversal of isoflurane anesthesia in rats as measured by cross-approximate entropy of the electroencephalogram. Anesthesiology 99:1125-1131. DOI 10.1097/00000542-200311000-00019

Jaušovec N, and Jaušovec K. 2010. Resting brain activity: differences between genders. Neuropsychologia 48:39183925. DOI 10.1016/j.neuropsychologia.2010.09.020

Katoh T, Suzuki A, and Ikeda K. 1998. Electroencephalographic derivatives as a tool for predicting the depth of sedation and anesthesia induced by sevoflurane. Anesthesiology 88:642-650. DOI 10.1097/00000542199803000-00014

Kelly SJ, Ostrowski NL, and Wilson MA. 1999. Gender differences in brain and behavior: hormonal and neural bases. Pharmacology Biochemistry and Behavior 64:655-664. DOI 10.1016/S0091-3057(99)00167-7

Kemali M, and Braitenberg V. 1969. Atlas of the Frog's Brain.

Koskinen M, Seppanen T, Tong S, Mustola S, and Thakor NV. 2006. Monotonicity of approximate entropy during transition from awareness to unresponsiveness due to propofol anesthetic induction. IEEE Transactions on Biomedical Engineering 53:669-675. DOI 10.1109/TBME.2006.870230

Kreuzer M, Hentschke H, Antkowiak B, Schwarz C, Kochs EF, and Schneider G. 2010. Cross-approximate entropy of cortical local field potentials quantifies effects of anesthesia-a pilot study in rats. BMC Neuroscience 11:122. DOI 10.1186/1471-2202-11-122

Laberge F, Muhlenbrock-Lenter S, Dicke U, and Roth G. 2008. Thalamo-telencephalic pathways in the fire-bellied toad Bombina orientalis. Journal of Comparative Neurology 508:806-823. DOI 10.1002/cne.21720

Lalonde-Robert V, Beaudry F, and Vachon P. 2012. Pharmacologic Parameters of MS222 and Physiologic Changes in Frogs (Xenopus laevis) After Immersion at Anesthetic Doses. Journal of the American Association for Laboratory Animal Science 51:464-468

Lalonde-Robert V, Desgent S, Duss S, and Vachon P. 2012. Electroencephalographic and physiologic changes after tricaine methanesulfonate immersion of African clawed frogs (Xenopus laevis). Journal of the American Association for Laboratory Animal Science 51:622-627

Li J, Yan J, Liu X, and Ouyang G. 2014. Using Permutation Entropy to Measure the Changes in EEG Signals During Absence Seizures. Entropy 16:3049-3061. DOI 10.3390/e16063049

Li X, Cui S, and Voss LJ. 2008. Using permutation entropy to measure the electroencephalographic effects of sevoflurane. Anesthesiology 109:448-456. DOI 10.1097/ALN.0b013e318182a91b

Liang Z, Wang Y, Sun X, Li D, Voss LJ, Sleigh JW, Hagihira S, and Li X. 2015. EEG entropy measures in anesthesia. Frontiers in Computational Neuroscience 9:16. DOI 10.3389/fncom.2015.00016

Lippolis G, Bisazza A, Rogers LJ, and Vallortigara G. 2002. Lateralisation of predator avoidance responses in three species of toads. Laterality 7:163-183. DOI 10.1080/13576500143000221

Llinas R, Ribary U, Contreras D, and Pedroarena C. 1998. The neuronal basis for consciousness. Philosophical Transactions of the Royal Society of London B Biological Sciences 353:1841-1849. DOI 10.1098/rstb.1998.0336

Mahon P, Greene B, Greene C, Boylan G, and Shorten G. 2008. Behaviour of spectral entropy, spectral edge frequency $90 \%$, and alpha and beta power parameters during low-dose propofol infusion. British Journal of Anaesthesia 
101:213-221. DOI 10.1093/bja/aen161

McCormick DA, and Bal T. 1997. Sleep and arousal: thalamocortical mechanisms. Annual Review of Neuroscience 20:185-215. DOI 10.1146/annurev.neuro.20.1.185

Merryman W, Boiman R, Barnes L, and Rothchild I. 1954. Progesterone "anesthesia" in human subjects. Journal of Clinical Endocrinology and Metabolism 14:1567-1569. DOI 10.1210/jcem-14-12-1567

Muthuswamy J, Roy R, and Sharma A. 1996. A study of electroencephalographic descriptors and end-tidal concentration in estimating depth of anesthesia. Journal of Clinical Monitoring and Computing 12:353-364. DOI 10.1007/BF02077633

Noh GJ, Kim KM, Jeong YB, Jeong SW, Yoon HS, Jeong SM, Kang SH, Linares O, and Kern SE. 2006. Electroencephalographic approximate entropy changes in healthy volunteers during remifentanil infusion. Anesthesiology 104:921-932. DOI 10.1097/00000542-200605000-00006

Ojemann GA. 1977. Asymmetric function of the thalamus in man. Annals of the New York Academy of Sciences 299:380-396. DOI 10.1111/j.1749-6632.1977.tb41923.x

Ouyang G, Dang C, Richards DA, and Li X. 2010. Ordinal pattern based similarity analysis for EEG recordings. Clinical Neurophysiology 121:694-703. DOI 10.1016/j.clinph.2009.12.030

Paduano M, Colafrancesco KC, Wong SA, Caldwell MS, and Gridi-Papp M. 2013. The Response of Gray Treefrogs to Anesthesia by Tricaine Methanesulfonate (TMS or MS-222). Isrn Zoology 2013:635704. DOI $10.1155 / 2013 / 635704$

Péran P, Cherubini A, Luccichenti G, Hagberg G, Démonet JF, Rascol O, Celsis P, Caltagirone C, Spalletta G, and Sabatini U. 2009. Volume and iron content in basal ganglia and thalamus. Human Brain Mapping 30:26672675. DOI 10.1002/hbm.20698

Pincus SM. 1991. Approximate entropy as a measure of system complexity. Proceedings of the National Academy of Sciences of the United States of America 88:2297-2301. DOI 10.1073/pnas.88.6.2297

Pincus SM. 1995a. Approximate entropy (ApEn) as a complexity measure. Chaos: An Interdisciplinary Journal of Nonlinear Science 5:110-117. DOI 10.1063/1.166092

Pincus SM. 1995b. Quantifying complexity and regularity of neurobiological systems. Methods in Neurosciences 28:336-363. DOI 10.1016/S1043-9471(06)80040-6

Pocock G, and Richards C. 1993. Excitatory and inhibitory synaptic mechanisms in anaesthesia. British Journal of Anaesthesia 71:134-147. DOI 10.1093/bja/71.1.134

Pritz MB. 2016. Thalamic reticular nucleus in Caiman crocodilus: forebrain connections. Neuroscience Letters 627:65-70. DOI 10.1016/j.neulet.2016.05.046

Ramlochansingh C, Branoner F, Chagnaud BP, and Straka H. 2014. Efficacy of tricaine methanesulfonate (MS-222) as an anesthetic agent for blocking sensory-motor responses in Xenopus laevis tadpoles. PloS One 9:101606. DOI 10.1371/journal.pone.0101606

Ries CR, and Puil E. 1999. Mechanism of anesthesia revealed by shunting actions of isoflurane on thalamocortical neurons. Journal of Neurophysiology 81:1795-1801. DOI 10.1152/jn.1999.81.4.1795

Robins A, and Rogers LJ. 2006. Complementary and lateralized forms of processing in Bufo marinus for novel and familiar prey. Neurobiology of Learning and Memory 86:214-227. DOI 10.1016/j.nlm.2006.03.002

Rogers LJ. 2014. Asymmetry of brain and behavior in animals: its development, function, and human relevance. Genesis 52:555-571. DOI 10.1002/dvg.22741

Rogers LJ, Rigosi E, Frasnelli E, and Vallortigara G. 2013. A right antenna for social behaviour in honeybees. 
Scientific Reports 3:2045. DOI 10.1038/srep02045

Rogers LJ, and Vallortigara G. 2015. When and Why Did Brains Break Symmetry? Symmetry 7:2181-2194. DOI $10.3390 /$ sym 7042181

Rogers LJ, Vallortigara G, and Andrew RJ. 2013. Divided brains: the biology and behaviour of brain asymmetries: Cambridge University Press.

Rogers LJ, Vallortigara G, and Rogers. 2017. Lateralized Brain Functions: Springer.

Rosa-Salva O, Regolin L, Mascalzoni E, and Vallortigara G. 2012. Cerebral and behavioural asymmetries in animal social recognition. Comparative Cognition \& Behavior Reviews 7:110-138. DOI 10.3819/ccbr.2012.70006

Roussigné M, Blader P, and Wilson SW. 2012. Breaking symmetry: The zebrafish as a model for understanding leftright asymmetry in the developing brain. Developmental Neurobiology 72:269-281. DOI 10.1002/dneu.20885

Samara A, and Tsangaris GT. 2011. Brain asymmetry: both sides of the story. Expert Review of Proteomics 8:693703. DOI 10.1586/epr.11.62

Schiff ND. 2008. Central thalamic contributions to arousal regulation and neurological disorders of consciousness. Annals of the New York Academy of Sciences 1129:105-118. DOI 10.1196/annals.1417.029

Schmidt RS. 1976. Neural correlates of frog calling. Journal of Comparative Physiology A: Neuroethology, Sensory, Neural, and Behavioral Physiology 108:99-113. DOI 10.1007/BF02169043

Schultz TW, and Dawson DA. 2003. Housing and husbandry of Xenopus for oocyte production. Lab Animal 32:34. DOI 10.1038/5000213

Sherman SM, and Guillery R. 2002. The role of the thalamus in the flow of information to the cortex. Philosophical Transactions of the Royal Society of London B Biological Sciences 357:1695-1708. DOI 10.1098/rstb.2002.1161

Sleigh JW, Steyn-Ross DA, Steyn-Ross ML, Grant C, and Ludbrook G. 2004. Cortical entropy changes with general anaesthesia: theory and experiment. Physiological Measurement 25:921-934. DOI 10.1088/0967$3334 / 25 / 4 / 011$

Steriade M. 1996. Arousal: revisiting the reticular activating system. Science 272:225. DOI 10.1126/science. 272.5259 .225

Sullivan EV, Rosenbloom M, Serventi KL, and Pfefferbaum A. 2004. Effects of age and sex on volumes of the thalamus, pons, and cortex. Neurobiology of Aging 25:185-192. DOI 10.1016/S0197-4580(03)00044-7

Torreilles SL, McClure DE, and Green SL. 2009. Evaluation and refinement of euthanasia methods for Xenopus laevis. American Association for Laboratory Animal Science 48:512-516

Vallortigara G, and Rogers LJ. 2005. Survival with an asymmetrical brain: advantages and disadvantages of cerebral lateralization. Behavioral and Brain Sciences 28:575-588. DOI 10.1017/S0140525X05350102

Vallortigara G, Rogers LJ, Bisazza A, Lippolis G, and Robins A. 1998. Complementary right and left hemifield use for predatory and agonistic behaviour in toads. Neuroreport 9:3341-3344. DOI 10.1097/00001756199810050-00035

Vallortigara G, and Versace E. 2017. Laterality at the neural, cognitive, and behavioral levels. In: Call J, ed. $A P A$ Handbook of Comparative Psychology: Vol 1 Basic concepts, methods, neural substrate, and behavior. Washington DC: American Psychological Association, 557-577.

Ward LM. 2011. The thalamic dynamic core theory of conscious experience. Consciousness and Cognition 20:464486. DOI 10.1016/j.concog.2011.01.007 
Ward LM. 2013. The thalamus: gateway to the mind. Wiley Interdisciplinary Reviews: Cognitive Science 4:609-622. DOI 10.1002/wcs. 1256

Wilczynski W, and Endepols H. 2007. Central auditory pathways in anuran amphibians: the anatomical basis of hearing and sound communication. In: Narins PM, Feng AS, Fay RR, and Popper AN, eds. Hearing and sound communication in amphibians. New York: Springer, 221-249.

Woolley CS, and Schwartzkroin PA. 1998. Hormonal effects on the brain. Epilepsia 39:S2-S8. DOI 10.1111/j.15281157.1998.tb02601.x

Xie G, Deschamps A, Backman SB, Fiset P, Chartrand D, Dagher A, and Plourde G. 2011. Critical involvement of the thalamus and precuneus during restoration of consciousness with physostigmine in humans during propofol anaesthesia: a positron emission tomography study. British Journal of Anaesthesia 106:548-557. DOI 10.1093/bja/aer060

Xue F, Fang G, Yang P, Zhao E, Brauth SE, and Tang Y. 2015. The biological significance of acoustic stimuli determines ear preference in the music frog. Journal of Experimental Biology 218:740-747. DOI $10.1242 /$ jeb. 114694

Zahl IH, Kiessling A, Samuelsen OB, and Hansen MK. 2009. Anaesthesia of Atlantic cod (Gadus morhua) - Effect of pre-anaesthetic sedation, and importance of body weight, temperature and stress. Aquaculture 295:52-59. DOI 10.1016/j.aquaculture.2009.06.019

Zahl IH, Kiessling A, Samuelsen OB, and Hansen MK. 2011. Anaesthesia of Atlantic halibut (Hippoglossus hippoglossus) Effect of pre-anaesthetic sedation, and importance of body weight and water temperature. Aquaculture Research 42:1235-1245. DOI 10.1111/j.1365-2109.2010.02711.x

Zhou J, Liu X, Song W, Yang Y, Zhao Z, Ling F, Hudetz AG, and Li S-J. 2011. Specific and nonspecific thalamocortical functional connectivity in normal and vegetative states. Consciousness and Cognition 20:257-268. DOI 10.1016/j.concog.2010.08.003 


\section{Table $\mathbf{1}$ (on next page)}

Results of ANOVA for ApEn as a function of the factors "stage", "sex" and "brain area"

Note: The symbols ' > ' denote that approximate entropy (ApEn) values for the given condition on the left side of ' > ' are significantly larger than those on the right side, and no significant difference exists among the corresponding conditions on the same side of ' $>$ ' for each case. Abbreviations: $F$, the $F$ value from ANOVA; $\varepsilon$, the values of epsilon of the Greenhouse-Geisser correction; partial $\eta^{2}$, effect size for ANOVA; LSD, least-significant difference test; I, Stage I; II, Stage II; III, Stage III; NA, not applicable. *, $p<0.05 ; * *, p<$ 0.001 . 
Table 1 Results of ANOVA for ApEn as a function of the factors "stage", "sex" and

"brain area"

\begin{tabular}{llcccc}
\hline Factors & \multicolumn{1}{c}{$\mathrm{F}$} & $\varepsilon$ & $p$ & partial $\eta^{2}$ & LSD \\
\hline Stage & $F_{2,24}=25.127$ & NA & $0.000^{* *}$ & 0.677 & I, IV > III \\
\hline Sex & $F_{1,12}=5.033$ & NA & $0.045^{*}$ & 0.295 & Female $>$ Male \\
\hline Brain area & $F_{5,60}=2.373$ & NA & 0.050 & 0.165 & NA \\
\hline Stage * Sex & $F_{2,24}=2.315$ & NA & 0.120 & 0.162 & NA \\
\hline Brain area * Sex & $F_{5,60}=0.903$ & NA & 0.485 & 0.070 & NA \\
\hline Stage * Brain area & $F_{10,120}=2.554$ & 0.364 & 0.057 & 0.175 & NA \\
\hline Stage * Sex * Brain area & $F_{10,120}=1.396$ & NA & 0.190 & 0.104 & NA \\
\hline
\end{tabular}

Note: The symbols ' > ' denote that approximate entropy (ApEn) values for the given condition on the left side of ' $>$ ' are significantly larger than those on the right side, and no significant difference exists among the corresponding conditions on the same side of '>' for each case. Abbreviations: $F$, the $F$ value from ANOVA; $\varepsilon$, the values of epsilon of the Greenhouse-Geisser correction; partial $\eta^{2}$, effect size for ANOVA; LSD, least-significant difference test; I, Stage I; II, Stage II; III, Stage III; NA, not applicable. *, $p<0.05 ;{ }^{* *}, p<0.001$. 


\section{Table 2 (on next page)}

Results of ANOVA for PE as a function of the factors "stage", "sex" and "brain area"

Note: The symbols ' > ' denote that permutation entropy (PE) values for the given condition on the left side of ' > ' are significantly larger than those on the right side, and no significant difference exists among the corresponding conditions on the same side of ' $>$ ' for each case. Abbreviations: $F$, the $F$ value from ANOVA; Partial $\eta^{2}$, effect size for ANOVA; $\varepsilon$, the values of epsilon of the Greenhouse-Geisser correction; LSD, least-significant difference test; I, Stage I; III, Stage III; IV, Stage IV; LT, left telencephalon; RT, right telencephalon; LD, left diencephalon; RD, right diencephalon; LM, left mesencephalon; RM, right mesencephalon; NA, not applicable. *, $p<0.05 ; * *, p<0.001$. 
Table 2 Results of ANOVA for PE as a function of the factors "stage", "sex" and "brain

\begin{tabular}{lccccc}
\multicolumn{5}{c}{ area” } \\
\hline Factors & \multicolumn{1}{c}{$\mathrm{F}$} & $\varepsilon$ & $p$ & partial $\eta^{2}$ & LSD \\
\hline Stage & $F_{2,24}=10.489$ & NA & $0.001^{*}$ & 0.466 & I, IV > III \\
\hline Sex & $F_{1,12}=7.895$ & NA & $0.016^{*}$ & 0.397 & Female $>$ Male \\
\hline Brain area & $F_{5,60}=10.584$ & NA & $0.000^{* *}$ & 0.469 & RM $>$ LT, RT, LD, RD, LM \\
& & & & & LD, RD, LM $>$ LT \\
& & & & & RD $>$ RT \\
\hline Stage * Sex & $F_{2,24}=0.961$ & NA & 0.397 & 0.074 & NA \\
\hline Brain area * Sex & $F_{5,60}=0.512$ & NA & 0.766 & 0.041 & NA \\
\hline Stage * Brain area & $F_{10,120}=6.665$ & NA & $0.000^{* *}$ & 0.357 & See Table 3 \\
\hline Stage * Sex * Brain area & $F_{10,120}=1.331$ & NA & 0.222 & 0.100 & NA \\
\hline
\end{tabular}

Note: The symbols ' > ' denote that permutation entropy $(\mathrm{PE})$ values for the given condition on the left side of ' > ' are significantly larger than those on the right side, and no significant difference exists among the corresponding conditions on the same side of '>' for each case. Abbreviations: $F$, the $F$ value from ANOVA; Partial $\eta^{2}$, effect size for ANOVA; $\varepsilon$, the values of epsilon of the Greenhouse-Geisser correction; LSD, least-significant difference test; I, Stage I; III, Stage III; IV, Stage IV; LT, left telencephalon; RT, right telencephalon; LD, left diencephalon; RD, right diencephalon; LM, left mesencephalon; RM, right mesencephalon; NA, not applicable. ${ }^{*}, p<$ $0.05 ; * *, p<0.001$. 


\section{Table 3 (on next page)}

Results of simple effects analysis for PE as a function of the factors "stage" and "brain area"

Note: The symbols ' > ' denote that permutation entropy (PE) values for the given condition on the left side of ' $>$ ' are significantly larger than those on the right side, and no significant difference exists among the corresponding conditions on the same side of ' $>$ ' for each case. Abbreviations: $F$, the $F$ value from ANOVA; Partial $\eta^{2}$, effect size for ANOVA; $\varepsilon$, the values of epsilon of the Greenhouse-Geisser correction; LSD, least-significant difference test; I, Stage I;

III, Stage III; IV, Stage IV; LT, left telencephalon; RT, right telencephalon; LD, left diencephalon; RD, right diencephalon; LM, left mesencephalon; RM, right mesencephalon; NA, not applicable. *, $p<0.05 ; * *, p<0.001$. 
Table 3. Results of simple effects analysis for PE as a function of the factors "stage" and

\begin{tabular}{|c|c|c|c|c|c|}
\hline \multicolumn{6}{|c|}{ "brain area" } \\
\hline Factors & $F$ & $\varepsilon$ & $p$ & partial $\eta^{2}$ & LSD \\
\hline \multicolumn{6}{|l|}{ Stage } \\
\hline \multirow[t]{2}{*}{ I } & $\mathrm{F}_{5,65}=6.583$ & 0.480 & $0.003^{*}$ & 0.336 & $\mathrm{RM}>\mathrm{LT}, \mathrm{RT}, \mathrm{LD}, \mathrm{RD}, \mathrm{LM}$ \\
\hline & & & & & $\mathrm{LD}>\mathrm{LT}$ \\
\hline \multirow[t]{2}{*}{ III } & $\mathrm{F}_{5,65}=8.642$ & NA & $0.000 * *$ & 0.399 & $\mathrm{RM}>\mathrm{LT}, \mathrm{RT}, \mathrm{LD}, \mathrm{RD}, \mathrm{LM}$ \\
\hline & & & & & $\mathrm{RD}>\mathrm{LT}, \mathrm{RT}$ \\
\hline \multirow[t]{3}{*}{ IV } & $\mathrm{F}_{5,65}=13.395$ & NA & $0.000^{* *}$ & 0.507 & $\mathrm{RM}>\mathrm{LT}, \mathrm{RT}, \mathrm{LD}, \mathrm{RD}$ \\
\hline & & & & & $\mathrm{LM}>\mathrm{LT}, \mathrm{RT}, \mathrm{LD}$ \\
\hline & & & & & $\mathrm{LD}>\mathrm{LT}$ \\
\hline \multicolumn{6}{|c|}{ Brain area } \\
\hline LT & $\mathrm{F}_{2,26}=13.942$ & NA & $0.000^{* *}$ & 0.517 & $\mathrm{I}>\mathrm{IV}>\mathrm{III}$ \\
\hline RT & $\mathrm{F}_{2,26}=12.234$ & NA & $0.000^{* *}$ & 0.485 & $\mathrm{I}>\mathrm{IV}>\mathrm{III}$ \\
\hline LD & $F_{2,26}=10.603$ & NA & $0.000 * *$ & 0.449 & $\mathrm{I}>\mathrm{IV}>\mathrm{III}$ \\
\hline $\mathrm{RD}$ & $\mathrm{F}_{2,26}=5.926$ & NA & $0.008^{*}$ & 0.313 & $\mathrm{I}>\mathrm{III}$ \\
\hline LM & $F_{2,26}=9.289$ & NA & $0.001 *$ & 0.417 & I, IV $>$ III \\
\hline $\mathrm{RM}$ & $F_{2,26}=6.507$ & NA & $0.005^{*}$ & 0.334 & I, IV $>$ III \\
\hline
\end{tabular}

Note: The symbols ' > ' denote that permutation entropy (PE) values for the given condition on the left side of ' > ' are significantly larger than those on the right side, and no significant difference exists among the corresponding conditions on the same side of ' $>$ ' for each case. Abbreviations: $F$, the $F$ value from ANOVA; Partial $\eta^{2}$, effect size for ANOVA; $\varepsilon$, the values of epsilon of the Greenhouse-Geisser correction; LSD, least-significant difference test; I, Stage I; III, Stage III; IV, Stage IV; LT, left telencephalon; RT, right telencephalon; LD, left diencephalon; RD, right diencephalon; LM, left mesencephalon; RM, right mesencephalon; NA, not applicable. ${ }^{*}, p<$ $0.05 ; * *, p<0.001$. 


\section{Table 4 (on next page)}

Results of the correlation analysis between ApEn and the duration of a given stage

Note: Abbreviations: $r$, correlation coefficient; LT, left telencephalon; RT, right telencephalon;

$L D$, left diencephalon; RD, right diencephalon; LM, left mesencephalon; RM, right mesencephalon; I, Stage I ; II, Stage II; III, Stage III; IV, Stage IV. *, $p<0.05$. 
Table 4 Results of the correlation analysis between ApEn and the duration of a given stage

\begin{tabular}{|c|c|c|c|c|c|c|c|c|c|c|c|c|}
\hline & \multicolumn{2}{|c|}{ LT } & \multicolumn{2}{|c|}{ RT } & \multicolumn{2}{|c|}{ LD } & \multicolumn{2}{|c|}{$\mathrm{RD}$} & \multicolumn{2}{|c|}{ LM } & \multicolumn{2}{|c|}{ RM } \\
\hline & $r$ & $p$ & $r$ & $p$ & $r$ & $p$ & $r$ & $p$ & $r$ & $p$ & $r$ & $p$ \\
\hline Duration (II)vs. ApEn (I) & 0.446 & 0.110 & 0.525 & 0.054 & 0.424 & 0.131 & 0.534 & $0.049^{*}$ & 0.433 & 0.122 & 0.481 & 0.081 \\
\hline Duration (II) vs. ApEn (II) & -0.068 & 0.817 & 0.196 & 0.503 & -0.002 & 0.994 & 0.305 & 0.288 & 0.231 & 0.427 & 0.284 & 0.326 \\
\hline Duration (II) vs. ApEn (III) & 0.253 & 0.383 & -0.051 & 0.864 & 0.182 & 0.533 & -0.042 & 0.887 & 0.323 & 0.260 & -0.042 & 0.887 \\
\hline Duration (II) vs. ApEn (IV) & -0.042 & 0.887 & -0.046 & 0.876 & -0.222 & 0.446 & -0.305 & 0.288 & 0.112 & 0.703 & 0.029 & 0.923 \\
\hline Duration (III) vs. ApEn (I) & -0.077 & 0.794 & -0.165 & 0.573 & -0.112 & 0.703 & -0.231 & 0.427 & 0.002 & 0.994 & -0.191 & 0.513 \\
\hline Duration (III) vs. ApEn (II) & -0.248 & 0.392 & -0.204 & 0.483 & -0.499 & 0.069 & -0.266 & 0.358 & -0.433 & 0.122 & -0.310 & 0.281 \\
\hline Duration (III) vs. ApEn (III) & -0.380 & 0.180 & -0.591 & $0.026^{*}$ & -0.407 & 0.149 & -0.600 & $0.023^{*}$ & -0.442 & 0.114 & -0.552 & $0.041^{*}$ \\
\hline Duration (III) vs. ApEn (IV) & -0.090 & 0.759 & -0.495 & 0.072 & -0.051 & 0.864 & -0.609 & $0.021^{*}$ & -0.051 & 0.864 & -0.323 & 0.260 \\
\hline
\end{tabular}

Note: Abbreviations: $r$, correlation coefficient; LT, left telencephalon; RT, right telencephalon; LD, left diencephalon; RD, right diencephalon; LM, left mesencephalon; RM, right mesencephalon; I, Stage I; II, Stage II; III, Stage III; IV, Stage IV. * $p<0.05$. 


\section{Table 5 (on next page)}

Results of the correlation analysis between PE and the duration of a given stage

Note: Abbreviations: $r$, correlation coefficient; LT, left telencephalon; RT, right telencephalon;

$L D$, left diencephalon; RD, right diencephalon; LM, left mesencephalon; RM, right mesencephalon; I, Stage I; II, Stage II; III, Stage III; IV, Stage IV. *, $p<0.05$. 
Table 5 Results of the correlation analysis between PE and the duration of a given stage

\begin{tabular}{|c|c|c|c|c|c|c|c|c|c|c|c|c|}
\hline & \multicolumn{2}{|c|}{ LT } & \multicolumn{2}{|c|}{ RT } & \multicolumn{2}{|c|}{ LD } & \multicolumn{2}{|c|}{$\mathrm{RD}$} & \multicolumn{2}{|c|}{ LM } & \multicolumn{2}{|c|}{$\mathrm{RM}$} \\
\hline & $r$ & $p$ & $r$ & $p$ & $r$ & $p$ & $r$ & $p$ & $r$ & $p$ & $r$ & $p$ \\
\hline Duration (II)vs. PE (I) & 0.451 & 0.106 & 0.354 & 0.215 & 0.358 & 0.208 & 0.130 & 0.659 & 0.130 & 0.659 & -0.160 & 0.584 \\
\hline Duration (II) vs. PE (II) & 0.248 & 0.392 & 0.477 & 0.085 & 0.130 & 0.659 & 0.446 & 0.110 & 0.371 & 0.191 & 0.248 & 0.392 \\
\hline Duration (II) vs. PE (III) & 0.349 & 0.221 & -0.015 & 0.958 & 0.024 & 0.935 & -0.275 & 0.342 & 0.182 & 0.533 & -0.147 & 0.615 \\
\hline Duration (II) vs. PE (IV) & 0.081 & 0.782 & 0.138 & 0.637 & 0.200 & 0.493 & -0.011 & 0.970 & 0.130 & 0.659 & -0.191 & 0.513 \\
\hline Duration (III) vs. PE (I) & 0.046 & 0.876 & 0.086 & 0.771 & 0.218 & 0.455 & 0.046 & 0.876 & 0.134 & 0.648 & -0.007 & 0.982 \\
\hline Duration (III) vs. PE (II) & -0.187 & 0.523 & -0.530 & 0.051 & -0.380 & 0.180 & -0.670 & $0.009^{*}$ & -0.455 & 0.102 & -0.543 & $0.045^{*}$ \\
\hline Duration (III) vs. PE (III) & -0.521 & 0.056 & -0.578 & $0.030^{*}$ & -0.490 & 0.075 & -0.543 & $0.045^{*}$ & -0.495 & 0.072 & -0.675 & $0.008^{*}$ \\
\hline Duration (III) vs. PE (IV) & -0.231 & 0.427 & -0.468 & 0.091 & -0.130 & 0.659 & -0.407 & 0.149 & -0.143 & 0.626 & -0.477 & 0.085 \\
\hline
\end{tabular}

Note: Abbreviations: $r$, correlation coefficient; LT, left telencephalon; RT, right telencephalon; LD, left diencephalon; RD, right diencephalon; LM, left mesencephalon; RM, right mesencephalon; I, Stage I; II, Stage II; III, Stage III; IV, Stage IV. *, $p<0.05$. 


\section{Table 6(on next page)}

Means and standard deviations of the durations of Stages II and III for females and males

Note: This table does not include Stages I and IV because the durations of these two stages were constant, i.e. $30 \mathrm{~min}$. Abbreviations: II, the administration stage; III, the recovery stage. $*, p<0.05$. 
Table 6 Means and standard deviations of the durations of Stages II and III for females and males

\begin{tabular}{lccc}
\hline Stages & Female (min) & Male (min) & $p$ \\
\hline II & $6.25 \pm 1.14$ & $4.78 \pm 1.03$ & $0.025^{*}$ \\
\hline III & $99.47 \pm 59.14$ & $139.33 \pm 53.95$ & 0.142 \\
\hline
\end{tabular}

Note: This table does not include Stages I and IV because the durations of these two stages were constant, i.e. $30 \mathrm{~min}$. Abbreviations: II, the administration stage; III, the recovery stage. ${ }^{*}, p<$ 0.05 . 
Figure 1

Electrode placements and $10 \mathrm{~s}$ of typical artifact-free EEG tracings for each channel during Stage I.

The intersection of the three dashed lines in the head of $X$. laevis denotes the lambda (i.e. the vertex where the skull sutures intersect). Abbreviations: LT and RT, the left and right telencephalon; LD and RD, the left and right diencephalon; LM and RM, the left and right mesencephalon.
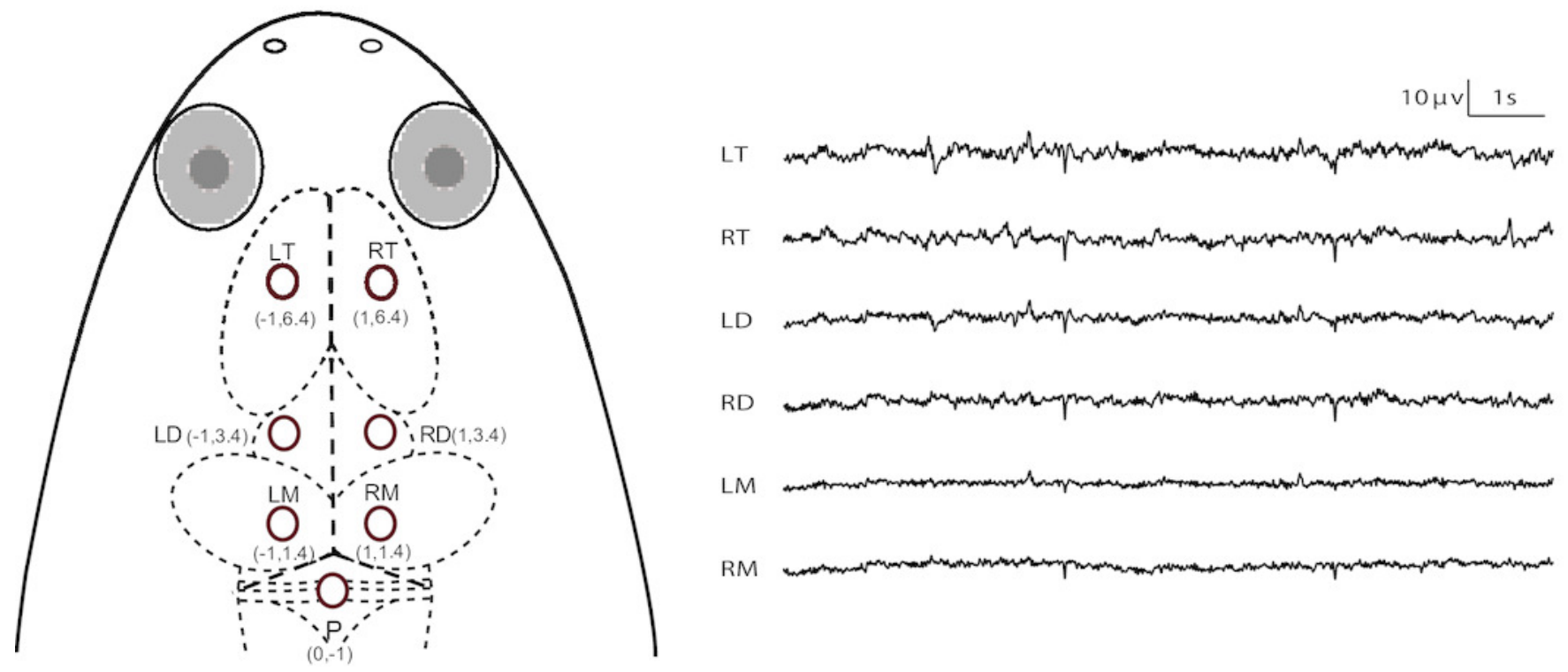


\section{Figure 2}

The dynamic variations of approximate entropy (ApEn) values $(A)$ and permutation entropy $(\mathrm{PE})$ values $(B)$ for the six brain regions for a randomly selected individual.

Note that epochs with artifact were not included. Since the time windows for ApEn (2 s) and PE (10 s) were different, the duration of a given artifact free epoch for the former was shorter than for the latter; thus, the durations of each stage for the former are longer than for the latter. Abbreviations: LT and RT, the left and right telencephalon; LD and RD, the left and right diencephalon; LM and RM, the left and right mesencephalon; I, Stage I (pre-anesthesia stage); II, Stage II (administration stage); III, Stage III (recovery stage); IV, Stage IV (postanesthesia stage).
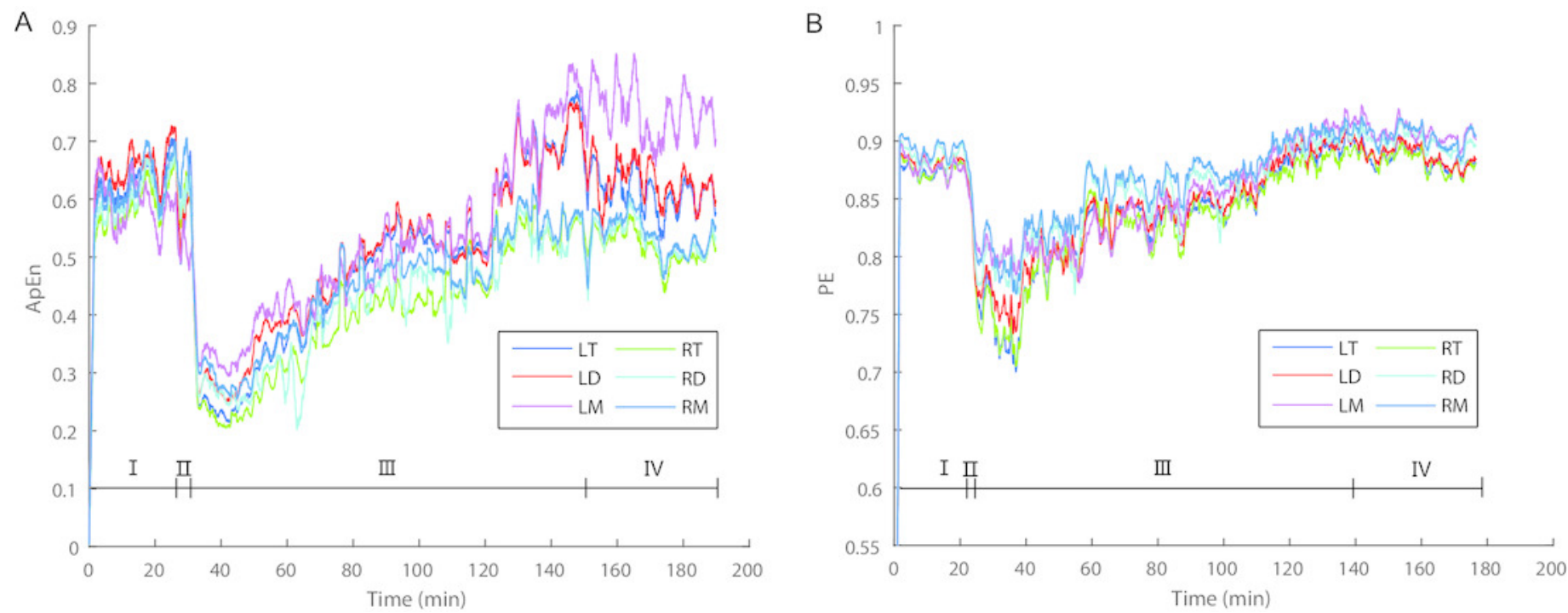
Figure 3

Correlation analysis between ApEn and the duration of a given stage for the right hemisphere

The correlation between the duration of Stage II and the approximate entropy (ApEn) values of brain structures during Stage I for the right hemisphere $(A-C)$, the correlation between duration of Stage III and the ApEn values of Stage III for the right hemisphere (D-F), and the correlation between the duration of Stage III and the ApEn values of Stage IV for the right hemisphere (G-I).
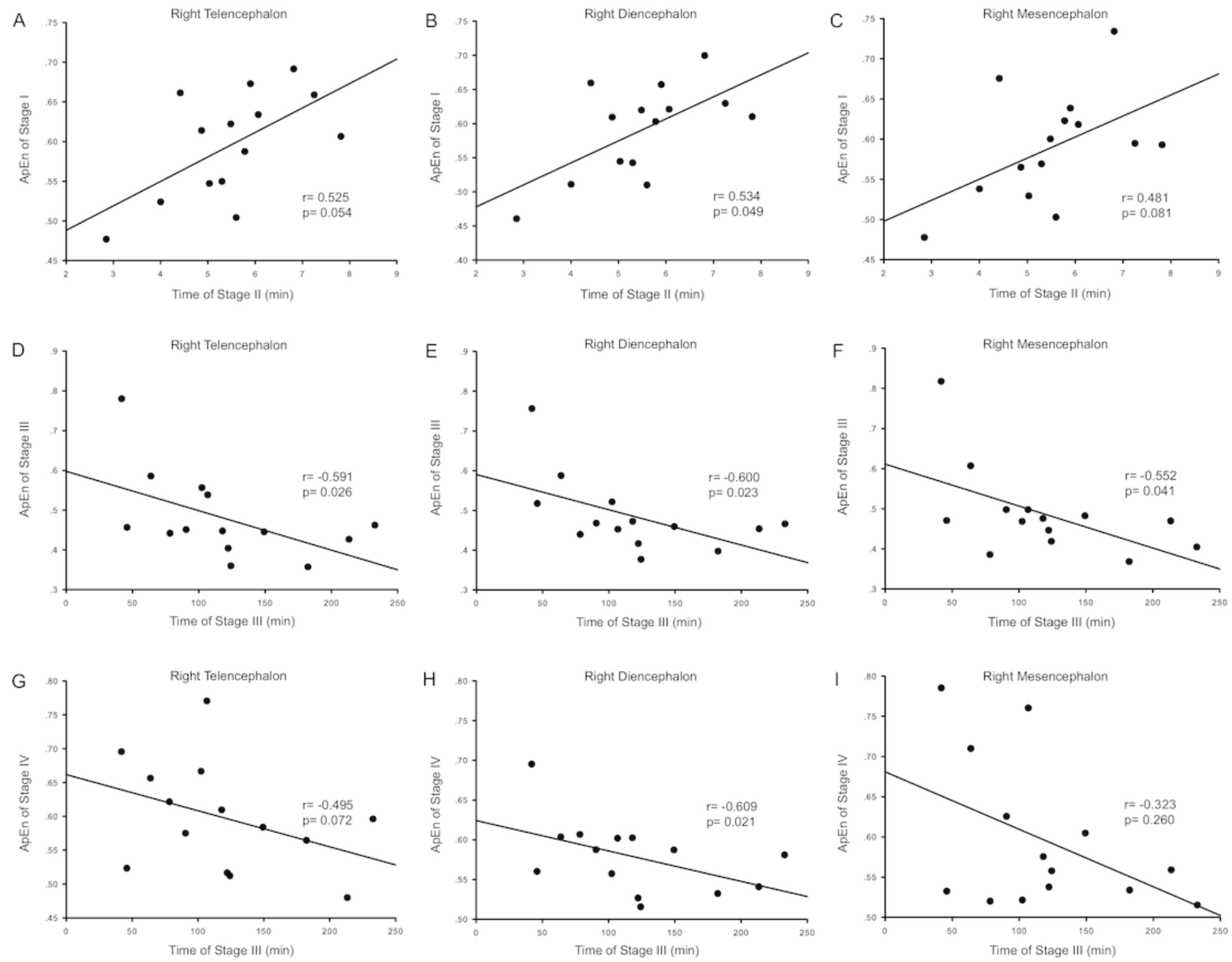


\section{Figure 4}

Correlation analysis between PE and the duration of a given stage for the right hemisphere

The correlation between the duration of Stage III and the Permutation entropy (PE) values of brain structures during Stage II for the right hemisphere (A-C), and the correlation between duration of Stage III and the PE values of Stage III for the right hemisphere (D-F).
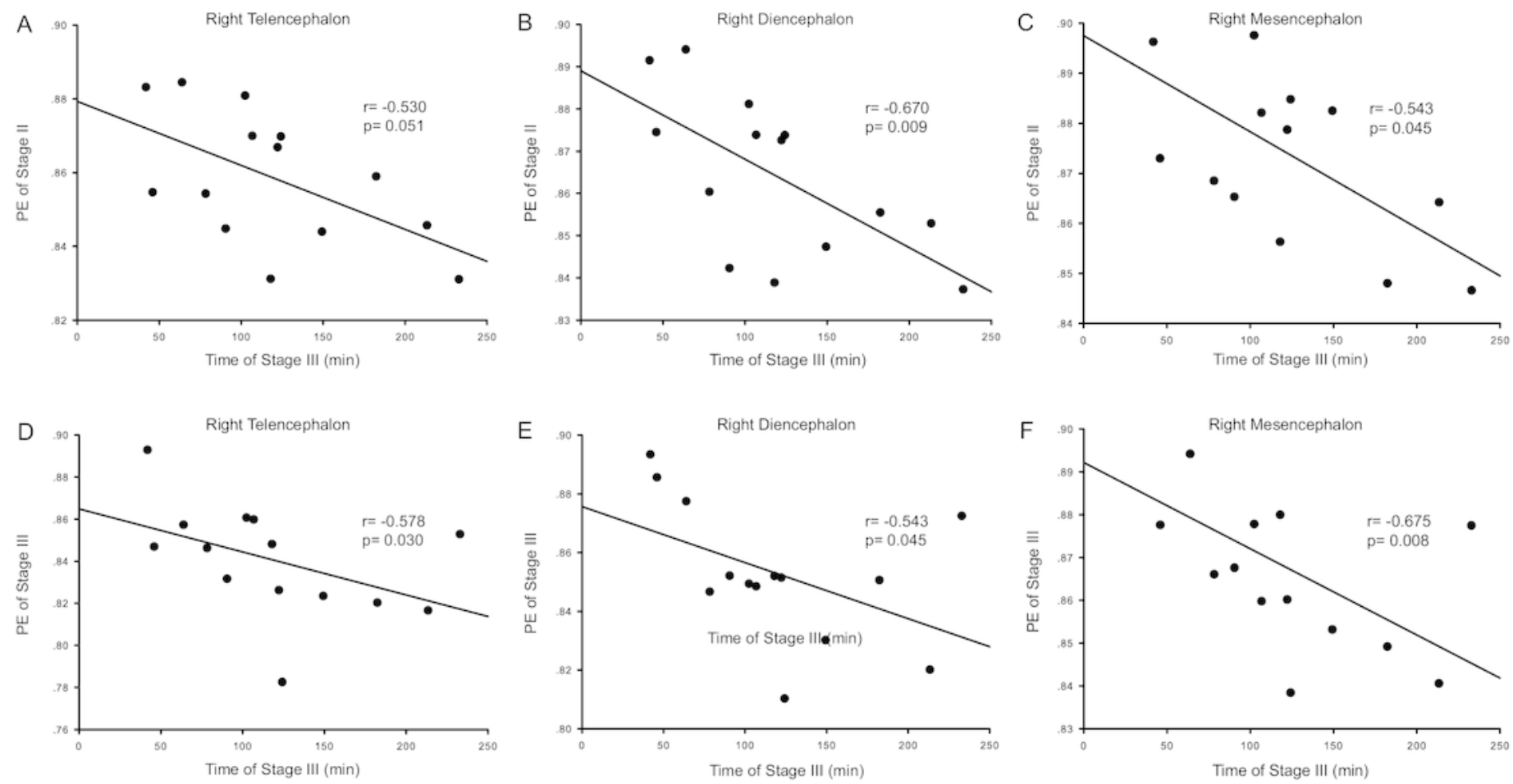